\title{
Orion Capsule Parachute Assembly System (CPAS) Main and Drogue Canopy Instrumentation
}

\author{
Jared S. Daum ${ }^{1}$ \\ NASA Johnson Space Center, Houston, Texas, 77059
}

\begin{abstract}
Reefing systems are often used in parachute systems to control loading and inflation of the canopies. These systems typically use pyrotechnic cutters to sever lines to progress through the staging. Existing cutters have been used for more than $\mathbf{5 0}$ years and have been proven adequate through countless demands. These cutters must survive and operate through severe environments that include a large snatch load at deployment and random vibration through the stage. In lieu of extensive drop test campaigns, future cutters will need to be ground tested to environments which are representative of the flight environments. The Capsule Parachute Assembly System (CPAS) developed sensors that capture accelerations and rates in three axes during parachute deployment and operation. These sensors have mass properties, size, and an attachment method similar to existing reefing line cutter assemblies, maximizing the usability of the data. Various risk reduction steps were taken to minimize any negative effects to the qualification parachute system and to increase the likelihood of collecting usable data. 24 sensors were flown on 12 qualification parachutes, successfully collecting sufficient data to characterize the deployment and operational environment of the reefing line cutters.
\end{abstract}

\section{Background}

The Capsule Parachute Assembly System (CPAS) utilizes 11 parachutes to safely slow the Orion spacecraft to a safe vertical velocity for touchdown. The deployment sequence is illustrated in Fig. 1. Three Forward Bay Cover Parachutes (FBCP) are mortar-deployed, providing sufficient force to ensure that the Forward Bay Cover (FBC) translates away from the Crew Module (CM) through the wake, mitigating the risk of recontact. Two Drogue parachutes are then mortar-deployed to slow and stabilize the CM for Main parachute deployment. Following the Drogues, three Main parachutes are deployed by individual mortar-deployed Pilot parachutes, decelerating the Orion $\mathrm{CM}$ to a speed safe for splashdown. Both the Main and Drogue parachutes utilize reefing stages to manage the canopy loading and organize inflation. Pyrotechnic cutters are used to sever the reefing lines, allowing the parachute to inflate to the next stage.

\footnotetext{
${ }^{1}$ CPAS Hardware Lead, Aeroscience and Flight Mechanics Division, NASA/JSC
} 


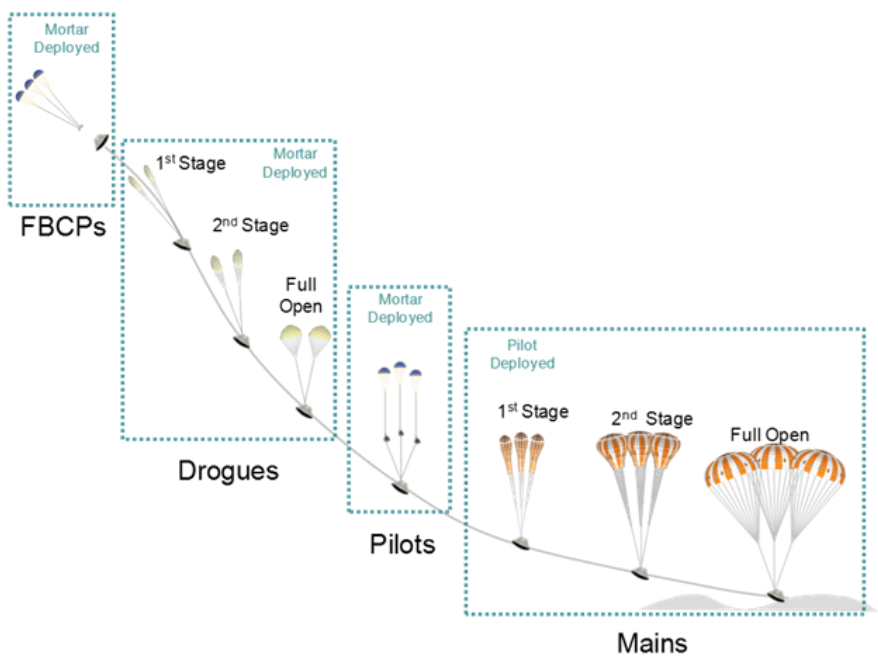

Fig. 1 Orion parachute deployment sequence.

\section{Motivation}

Current pyrotechnic reefing line cutters (RLC) are procured by NASA using a procurement specification which describes the operational limits, weight, and cutting capacity, among other characteristics of the cutter. Due to the design of existing cutters, the operational dynamic environment is of particular interest as large accelerations could prohibit the actuation of the RLC or otherwise degrade the performance of the device. Currently, this specification does not define the peak acceleration or random vibration environment that a cutter must endure. This has been acceptable to the industry due to the long history of successful use of these particular cutters. In order to for a current or future program to accept a new cutter design or vendor, the acceleration and vibration environments should be defined and tested to in lieu of extensive operational history. It is of interest to CPAS to define the operational environment of an RLC in the required flight envelope with the parachutes in the flight configuration. With qualification testing quickly coming to an end (approximately five drop tests remaining), the CPAS team set out to attempt to define this environment.

\section{Approach and Design}

The CPAS team determined that the most appropriate approach to collecting this data, given the remaining drop test schedule, was to design a sensor which mimics the properties of the existing RLC plus structural sleeve. Installing sensors with similar mass, inertia, and dimensions into the canopy, in a similar manner as the RLC, would yield data that is directly relatable to the environment seen by the RLC. Although slightly larger in diameter and length, and thus volume, the aluminum housing and printed circuit board shape of the RLCES results in an approximately equal mass to that of the cutters. The slightly longer length was deemed acceptable.

\section{Fig. 2 Dimensional comparison of RLCES (top) and RLC variants flown in CPAS testing (bottom).}

The sensor was designed to include a 3-axis accelerometer, 3-axis gyroscope, battery, onboard memory, and other various components needed to collect the desired data.

Of particular concern to the parachute community is the interface of the sensor to the canopy. This is manifested in both the anchoring of the sensor to the skirt and the method by which the sensor is activated. In order to collect data that are most representative of actual RLC assemblies, the sensor was attached to the canopy using a pocket nearly identical to that of the cutter pocket, but on the outside of the canopy rather than the inside to avoid interfering with the reefing system. To minimize potential impact to the surrounding parachute, a textile pull lanyard activation method was developed. The RLCES components include a spring-loaded plunger type switch which is held in an open state 
by a Spectra lanyard anchored low on the parachute riser in a "lazy-leg" configuration. Upon deployment of the parachute, the Spectra lanyard is stripped out of the sensor, allowing the plunger contacts to close and thus power the system. A visualization of the anchoring, packed configuration, and deployed configuration of the actuating lanyard is seen in Fig. 3. The lanyard is connected to the loop to loop interface with an additional loop of Spectra in order to minimize any impact to the qualification system.
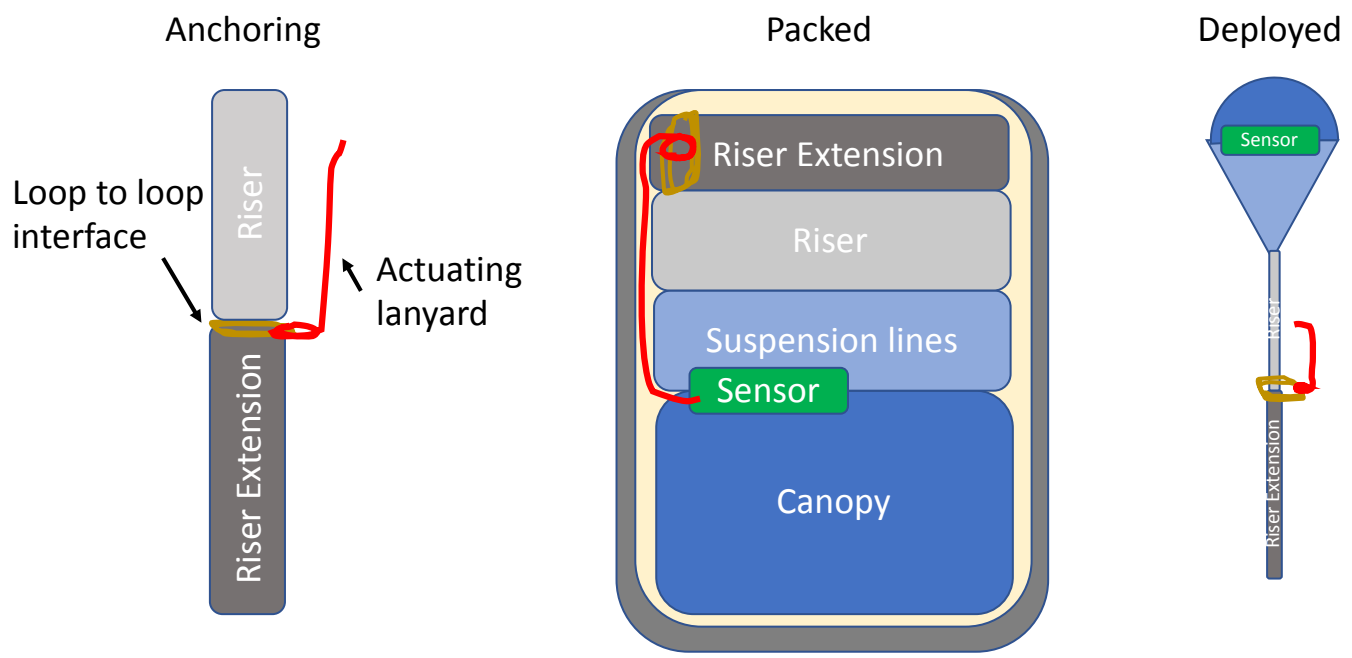

Fig. 3 Anchoring, packed configuration, and deployed configuration of the actuating lanyard.

\section{Risk Reduction}

The risks introduced to a system in qualification by adding experimental instrumentation were not lost on the CPAS team. Deploying point masses in the skirt of a canopy is cause for concern for parachute designers. Additionally, adding hardware or instrumentation during qualification that is not part of the final configuration should be avoided. The project determined the risk incurred by introducing the sensors in the qualification system was acceptable, particularly when compared with potential future risks the human-rated system might face if required to accept future cutters without quantifying these environments or executing an extensive drop test campaign. To mitigate the risk of flying the sensors on the qualification parachutes, the CPAS project undertook two risk reduction paths as outlined in the following sections. While the primary objective of these risk mitigations was to identify and address any potential impact the sensor could have to the parachutes, a secondary objective was to gain demands of the actuation and operation of the sensors to increase the likelihood of gathering data in the qualification parachutes.

\section{A. Implementation on Test Support Parachutes}

One method of risk reduction was to implement the sensors in two types of test support parachutes during qualification drop tests. The programmer parachutes that stabilize the vehicle and put it at the test point, seen in Fig. 4 , are nearly identical to the qualification Drogue parachutes, although they are static line deployed rather than mortardeployed. The recovery Main parachutes, also seen in Fig. 4, are used to safely land the pallet from which the test vehicle is extracted, and are nearly identical to the qualification Main parachutes. While it is understood that this data may not be applicable to the qualification system, at a minimum these parachutes would provide experience in the operation of the sensors. 


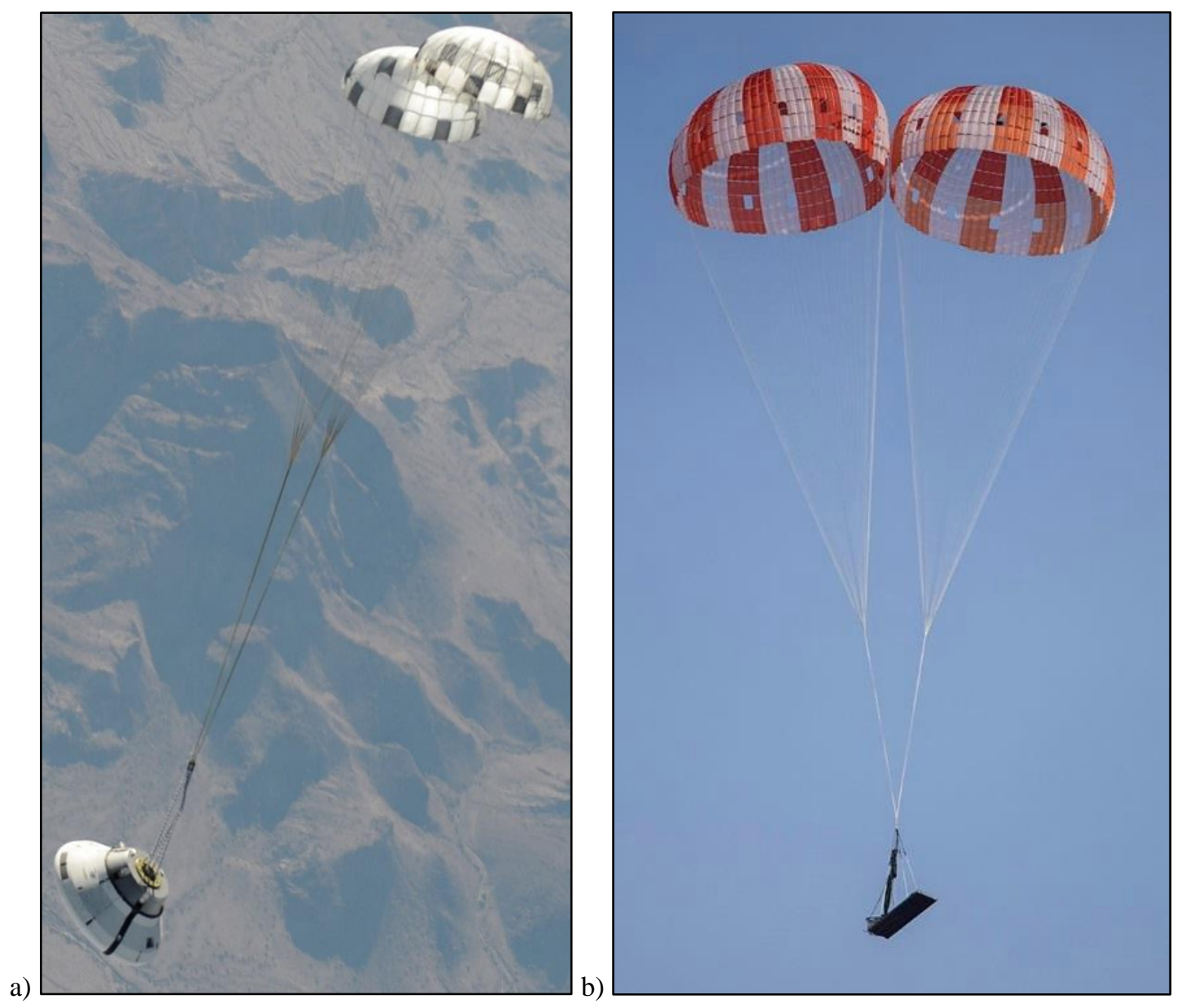

Fig. 4 a) CPAS drop test vehicle under programmer parachutes. b) CPAS pallet under recovery mains

Preliminary designs of the sensors were flown on two recovery parachutes and six programmer parachutes on qualification tests 4-3 through 4-5 totaling 18 sensor demands. The design of the sensor components and housing evolved through these tests while the general shape and mass remained similar to the RLC. No noticeable impacts to the parachutes' deployment or performance were attributed to the sensors after thorough video and data reviews. Detailed inspection of the parachutes after testing revealed no significant damage that would indicate a snag or other hazard to the parachute. This provided evidence that including the sensors may have minimal impact to the qualification configuration.

\section{B. Other Risk Mitigation Steps}

In addition to flying the sensors in the test support parachutes, four risk mitigation activities were performed. Project and Orion Program personnel agreed that successful completion of these activities was sufficient evidence that the qualification test would not be compromised if the sensor is included.

\section{Simulator Lanyards}

The activation method for the RLCES was designed for minimal impact to the qualification parachutes. Flightlike packing and integration of the Main and mortar-deployed Drogue parachutes, along with snag hazard concerns, required the activation method be completely self-contained inside the deployment bag. Additionally, it was desired to activate the sensors as early as possible in the deployment so that the system could boot up and begin collecting data as early as possible. This required a length of the Spectra activation lanyard to be pulled past parachute material. Simulator lanyards (with no sensors) were installed in the qualification Main and Drogue parachutes on CQT4-5 (Cluster Qualification Test), as seen in the Drogue in Fig. 5. The primary test objectives were to observe any damage 
to the qualification assets that could be attributed to the lanyards and to verify that the lanyard pull method would not result in broken lanyards or any other conditions that would prevent sensor activation.

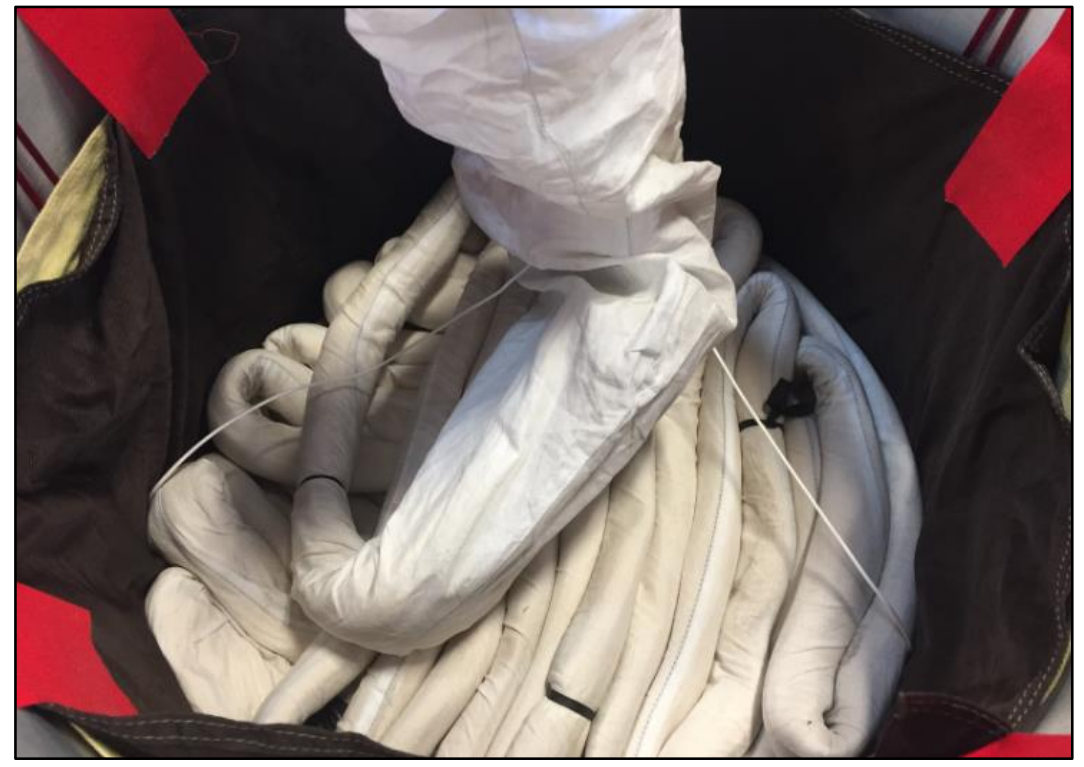

Fig. 5 Sensor activation lanyard in Drogue parachute.

All 8 simulator lanyards performed as expected. The entire length of installed lanyard remained fixed to the attach point at post-test inspection. Additionally, there was no indication of friction burns or other damage to the qualification asset. This provided confidence that the sensors could be activated without impacting the qualification parachutes.

\section{Thermal Vacuum Testing and Analysis}

CPAS Main parachutes undergo autoclave at approximately 200 degrees $F$ for 48 hours. The purpose of this testing was to demonstrate that the sensor components (primarily the battery) and housing could survive the autoclave environment without affecting the parachute, whether that is damage/leaking from the battery, or mechanical interferences causing sharp edges. Initial testing was conducted at 201.5 degrees $\mathrm{F}$ for 48 hours. Minimal changes in battery size and performance were noted. Additionally, analysis showed large margins on the battery shell with sufficient clearance to the inside of the housing under thermal vacuum conditions. Due to high confidence in the understanding of these batteries in thermal vacuum environments, NASA pyrotechnics does not require additional vacuum testing on cylindrical cells such as these.

\section{X-ray Testing}

CPAS Main and Drogue parachutes undergo X-ray testing during the packing process for various reasons, including screening for Foreign Object Debris (FOD) or bent RLC assemblies. As a risk reduction to the sensors ability to collect data, a RLCES assembly was tested under similar X-ray conditions. The sensor was configured for testing, ground tested to confirm read/write, subjected to X-ray, and then ground tested again. Test results showed that the X-ray conditions did not impact sensor performance.

\section{Seam and Joint Testing}

Stitching, knotting, joining, or otherwise affecting raw textile materials can degrade the ultimate strength of that material. Introduction of the RLCES into the canopies required the addition of custom pockets which were sewn into the parachute. Testing was required to demonstrate that joining the pocket materials to the parachute structural grid did not significantly degrade the structural capability of the parachute. Since these pockets are slightly different from the flight cutter pockets (located on the outside of the canopy), samples were pull tested, as seen in Fig. 6, and results showed that the addition of the pockets did not significantly degrade the parachutes. 


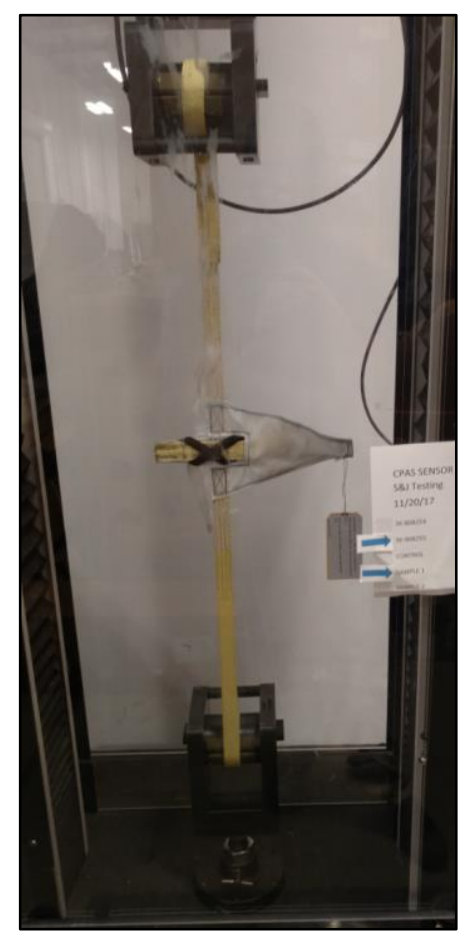

Fig. 6 Sensor pocket seam and joint testing.

\section{Drop Testing and Results}

With all of the risk mitigation steps completed, the project proceeded to fly the sensor in all available qualification Main and Drogue parachutes on tests CQT4-6, CQT4-7, and CQT4-8. In total, 24 sensors were flown and 16 of those successfully collected data. Eight of the sensors failed to record useful data for various reasons including shorting of the electrical system to the housing, damaged components, and other unknown issues. Fig. 7 shows an example time history of the three-axis accelerations measured on a Main parachute for CQT4-6, which was a deployment at a dynamic pressure of approximately 110 pounds per square foot.

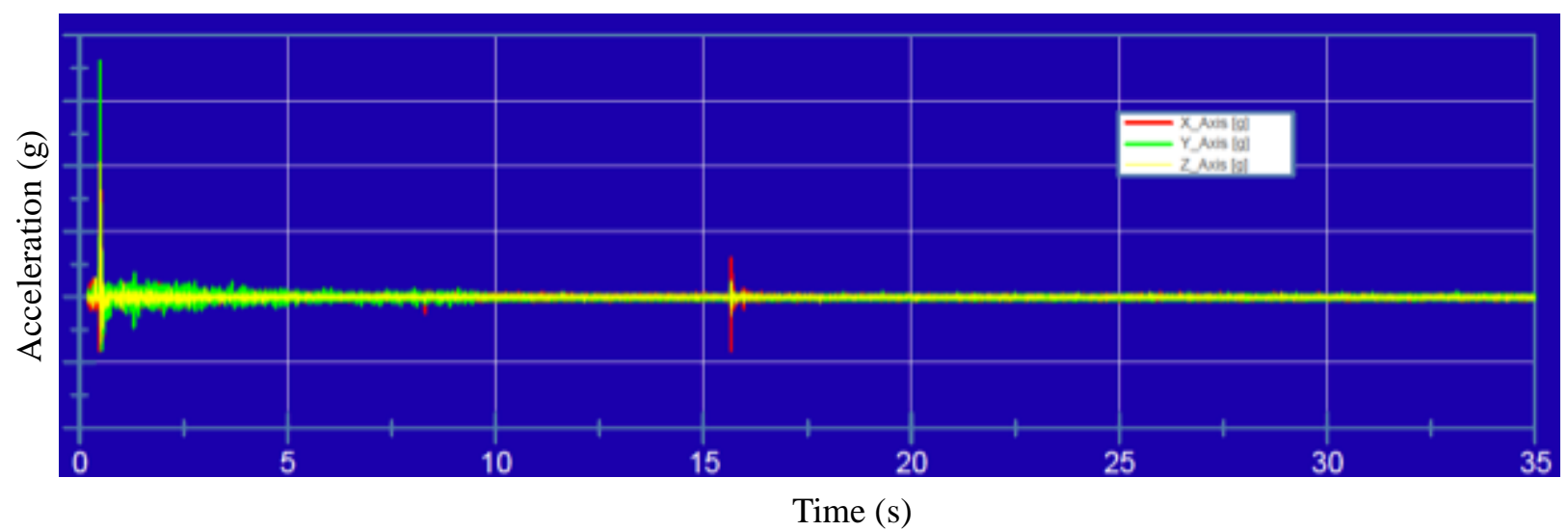

Fig. 7 Main acceleration data from CQT4-7.

As expected, a large acceleration spike is seen at the beginning of the deployment which correlates to the snatch of the sensor from a stowed position inside the deployment bag to the deployed position on the tensioned suspension line. Additionally, high frequency oscillations are seen after the snatch load which indicate that skirt of the parachute is fluttering in the high dynamic pressure. This flutter is significantly reduced at around the 9 second mark, which corresponds to disreef of the parachute to second stage ( 8 second cutter plus 1 second of sensor activation before cutter 
activation). Lastly, a transient acceleration signature is seen near the 16 second mark. This is at the $2^{\text {nd }}$ stage disreef point (16 second cutter) and may indicate that the end of the reefing line contacted the sensor as it is whipping through the canopy. While this signature is not of particular concern, it is indeed interesting. Fig. 8 shows rate data from the 3 -axis gyroscope. Similar signatures as describe in the accelerometers can be seen.

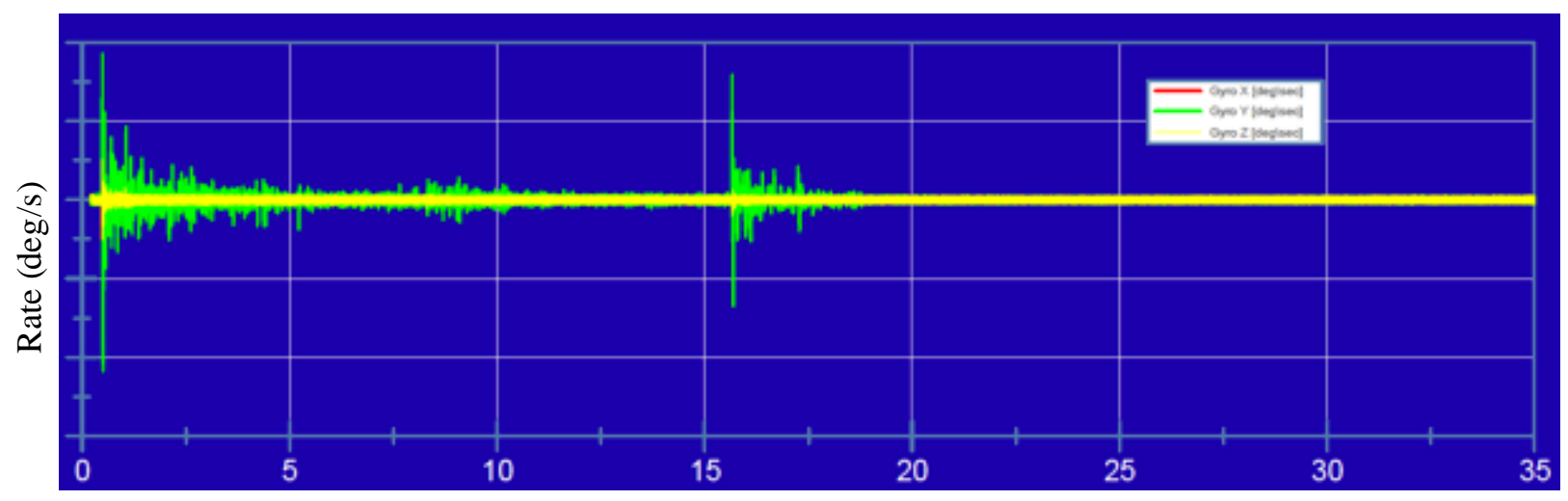

Time (s)

Fig. 8 Main rate data from CQT4-7.

Acceleration data from one of the sensors on a Drogue parachute from CQT4-7 is seen in Fig. 9. The Drogues were mortar-deployed at a high dynamic pressure of approximately 160 pounds per square foot. Similar signatures as described in the Main parachutes can be seen, although the large signatures at disreef are not apparent. The staging (particularly at 16 seconds) is marked by a noticeable decrease in vibration signature, indicating that flutter of the skirt is decreasing in magnitude.

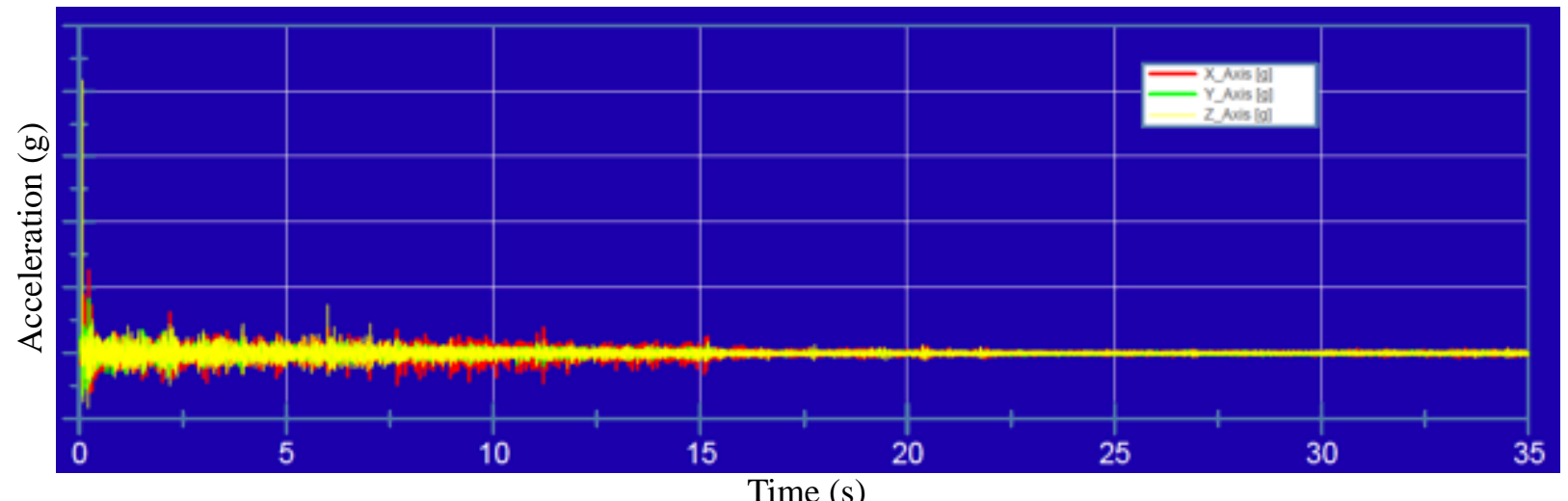

Fig. 9 Drogue acceleration data from CQT4-7.

Of particular note is the proximity of the snatch load with the first data points collected by the sensor. Taking a closer look, Fig. 10 shows that the sensor was indeed booted in time to catch the snatch event as there are approximately 0.075 seconds of data before the transient signature. This is not unexpected as the Drogues are mortardeployed at approximately 140 feet per second, with line sail further reducing the time between sensor lanyard pull and snatch load. This is in contrast to the Main parachutes which are pilot-deployed and travel approximately 220 feet before snatch load, providing enough time for the sensor to boot and begin collecting data before snatch loading occurs. 


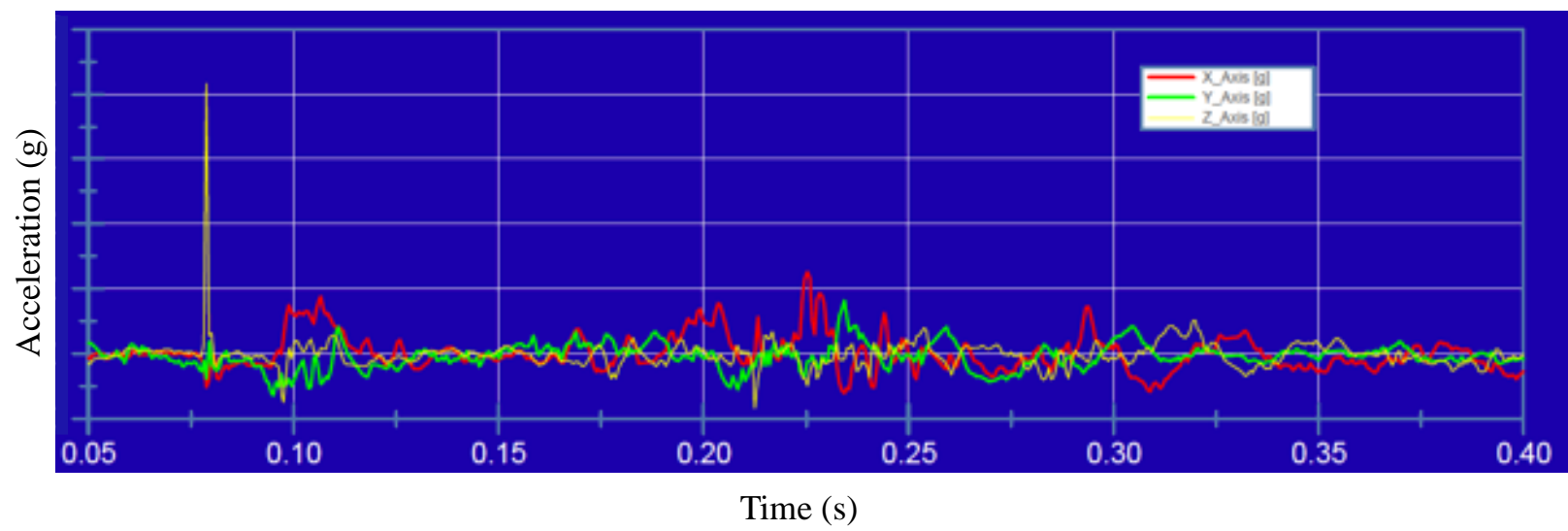

Fig. 10 Snatch load in acceleration data from CQT4-7 Drogue.

Unfortunately, the Drogue sensor data shown above appears to be the only sensor in the qualification testing that captured the peak snatch acceleration. Fortunately, this was the single high dynamic pressure test, which is expected to have the highest snatch loads. Other sensors appear to have begun capturing data after the transient event due to unknown delays in activation and boot-up. One potential explanation is friction, jamming, or inertial effects increasing the time it takes for the plunger switch to close. Fig. 11 shows the rate data from a Drogue sensor on CQT4-7. Flutter in the first stage is evident by the large oscillations seen before the 8 second mark.

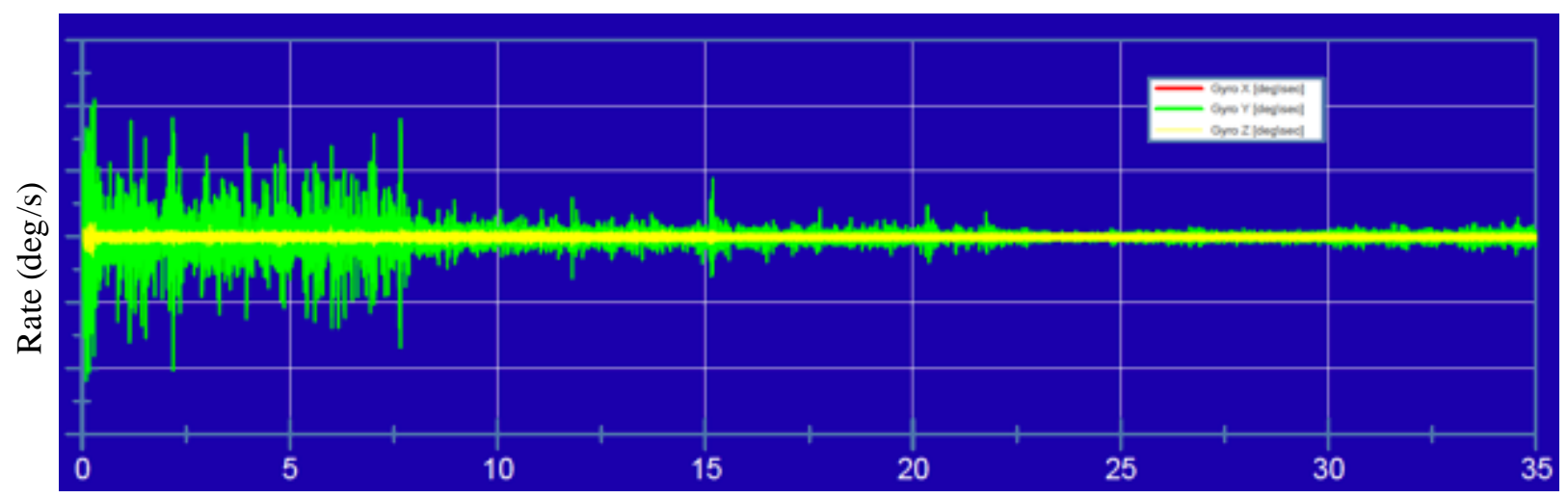

Time (s)

Fig. 11 Drogue rate data from CQT4-7.

\section{Conclusion}

Driven by the desire to be able to qualify future reefing line cutters for use on CPAS with assumed limited available testing, the team designed and implemented a sensor which successfully gathered data regarding the accelerations and rates at the skirt of the Main and Drogue canopies during qualification deployments, including CQT4-7 at bounding dynamic pressures. Although several sensors experienced malfunctions and issues with the boot time of the Drogue sensors was limited Drogue snatch load data, the sensors provided generally healthy and valid data that will support the development and acceptance of future reefing line cutters. 